\title{
Concentrating the dark matter in galaxy clusters through tidal stripping of baryonically compressed galactic halos
}

\author{
Rennan Barkana ${ }^{1,2 \star}$ and Abraham Loeb ${ }^{3 \star}$ \\ ${ }^{1}$ Division of Physics, Mathematics and Astronomy, California Institute of Technology, Mail Code 130-33, Pasadena, CA 91125, USA \\ ${ }^{2}$ Raymond and Beverly Sackler School of Physics and Astronomy, Tel Aviv University, Tel Aviv 69978, Israel \\ ${ }^{3}$ Astronomy Department, Harvard University, 60 Garden Street, Cambridge, MA 02138, USA
}

Accepted 2010 February 24. Received 2010 February 23; in original form 2009 July 6

\begin{abstract}
Gravitational lensing observations of massive X-ray clusters imply a steep characteristic density profile marked by a central concentration of dark matter. The observed mass fraction within a projected radius of $150 \mathrm{kpc}$ is twice that found in state-of-the-art dark matter simulations of the standard $\Lambda$ cold dark matter cosmology. A central baryon enhancement that could explain this discrepancy is not observed, leaving a major puzzle. We propose a solution based on the merger histories of clusters. A significant fraction of the final dark matter content of a cluster halo originates within galaxy-sized haloes, in which gas can cool and compress the dark matter core to high densities. The subsequent tidal stripping of this compressed dark matter occurs in denser regions that are closer to the centre of the cluster halo. Eventually, the originally cooled gas must be dispersed into the intracluster medium through feedback, for consistency with observations that do not find central baryon enhancements in clusters. Still, the early adiabatic compression of the galactic dark matter leaves a net effect on the cluster. Using a simple model for this process, we show that the central cluster profile is substantially modified, potentially explaining the observed discrepancy.
\end{abstract}

Key words: galaxies: clusters: general - galaxies: formation - dark matter - cosmology: theory.

\section{INTRODUCTION}

Recent observations have confirmed our basic understanding of cosmology and showed an impressive consistency with the predictions of the standard $\Lambda$ cold dark matter (CDM) model (e.g. Astier et al. 2006; Percival et al. 2007; Komatsu et al. 2009). In this model, a cosmological constant dominates the cosmic mass budget today, but galaxies and other structures were assembled earlier, primarily out of CDM. This medium of non-interacting, low velocity-dispersion particles, started out with small Gaussian density perturbations that were subsequently enhanced by gravity. While the model successfully matches observations of the large-scale anisotropies of the cosmic microwave background and the large-scale structure in galaxy surveys, it is also important to test its validity on smaller scales. The abundance and structure of non-linear objects are potentially sensitive probes of the properties of dark matter (e.g. whether it is cold) and of the density fluctuations (e.g. whether they are Gaussian). However, gas cooling and astrophysical feedback complicate the interpretation of observations regarding the dark matter distribution in galaxies. Thus, it is most attractive to study the largest virialized

^E-mail: barkana@wise.tau.ac.il (RB); aloeb@cfa.harvard.edu (AL) objects, namely X-ray clusters, in which most of the gas is too hot and rarefied to cool and is thus expected to trace the gravitational potential.

The mass profiles of galaxy clusters can be measured directly through gravitational lensing. Observations of the most massive clusters now find dozens of multiply imaged background sources, allowing a precise measurement of the central 2D mass distribution in each cluster as projected on the sky. Also crucial for characterizing each cluster is its total virial mass, which can be measured precisely by supplementing the central strong lensing signal with weak lensing distortions measured out to the cluster edge. It is useful to characterize the total, projected profile with one scale, the effective Einstein radius $r_{\mathrm{E}}$ (or angle $\theta_{\mathrm{E}}$ ) defined so that a circle of that radius around the cluster centre contains a mean enclosed surface mass density $\bar{\Sigma}$ equal to the critical density for lensing, $\Sigma_{\mathrm{cr}}=\left[c^{2} /(4 \pi G)\right] D_{\mathrm{OS}} /\left(D_{\mathrm{OL}} D_{\mathrm{LS}}\right)$, where $D$ denotes various angular diameter distances (Observer-Source, Observer-Lens and LensSource). This definition is motivated by the Einstein ring radius of an axisymmetric lens, but is none the less a useful measure of the central matter content even for asymmetric clusters.

Current $N$-body simulations of galaxy clusters in $\Lambda$ CDM produce samples of thousands of haloes with virial mass $M_{\text {vir }}>10^{14} \mathrm{M}_{\odot}$. These simulations are becoming sufficiently large and detailed to 
yield the predicted spread in cluster halo parameters, and to allow a quantitative assessment of the inherent bias in observing clusters in projection and selecting them by lensing cross-section (Hennawi et al. 2007; Neto et al. 2007). In general, the density profiles of the simulated clusters are relatively shallow and seemingly at odds with recent careful lensing studies of massive clusters (Gavazzi et al. 2003; Kneib et al. 2003; Broadhurst et al. 2005; Kling et al. 2005; Limousin et al. 2007; Bradač et al. 2008; Halkola et al. 2008; Umetsu \& Broadhurst 2008).

This discrepancy was recently highlighted and quantified by Broadhurst \& Barkana (2008), who carefully compared observations of four well-constrained massive clusters to the predictions of the numerical simulations. They emphasized the importance of comparing directly the projected 2D mass distributions in the observations and the simulations, using the virial mass $\left(M_{\mathrm{vir}}\right)$ and the effective Einstein radius $\left(r_{\mathrm{E}}\right)$ as two numbers that characterize the degree of concentration in each cluster halo. They derived the theoretical predictions for cluster lensing in $\Lambda \mathrm{CDM}$ by starting with the distribution of 3D halo profiles measured by Neto et al. (2007) in the Millennium simulation, and then correcting it for lensing and projection biases based on Hennawi et al. (2007). Comparing the resulting distribution with the observed $r_{\mathrm{E}}$ for four clusters $-\mathrm{A} 1689$, Cl0024, A1703 and RX J1347 - and including the expected spread in profiles as well as the measurement errors, they found that each cluster was discrepant at the $2 \sigma$ level (all with an unusually large $r_{\mathrm{E}}$, given $M_{\text {vir }}$ ), yielding a combined $4 \sigma$ discrepancy. Duffy et al. (2008) recently found that simulated cluster concentrations are even lower when using the most updated cosmological parameters (which have a lower power spectrum normalization than assumed by Neto et al. (2007)), though the effect for the most massive clusters is only at the level of $\sim 10$ per cent.

Broadhurst \& Barkana (2008) suggested that gas physics is unlikely to affect significantly the Einstein radius of a cluster. This radius of $\sim 150 \mathrm{kpc}$ is typically observed to enclose a projected mass of $\sim 2 \times 10^{14} \mathrm{M}_{\odot}$, or a mass of $\sim 1 \times 10^{14} \mathrm{M}_{\odot}$ within the same 3D radius. Using the simple model of adiabatic compression (Blumenthal et al. 1986), they estimated that the observed 3D mass within the Einstein radius can be obtained if gas cooling increases the enclosed baryonic fraction within this radius to $\sim 1 / 3$, twice the cosmic baryon fraction. Indeed, hydrodynamic simulations produce clusters that are as centrally concentrated as those observed, likely due to their 'overcooling' problem which produces just such an increase in the central baryon fraction, with most of it in stars (Kravtsov, Nagai \& Vikhlinin 2005; Rozo et al. 2008). An increase of this sort apparently does not occur in real clusters, where the baryonic (gas+stellar) mass fraction within the Einstein radius is below the cosmic value (e.g. Lin, Mohr \& Stanford 2004; LaRoque et al. 2006; Afshordi et al. 2007; Lemze et al. 2008; Vikhlinin et al. 2009). Thus, Broadhurst \& Barkana (2008) concluded that cluster halo profiles present perhaps the clearest, most robust, current conflict between observations and the standard $\Lambda$ CDM model. Subsequent work has generally supported this conclusion (e.g. Oguri \& Blandford 2009; Oguri et al. 2009; Zitrin \& Broadhurst 2009), though only a large unbiased cluster sample with precise strong and weak lensing measurements would be completely conclusive.

In this paper, we propose a novel process that could resolve the apparent discrepancy between cluster observations and existing $\Lambda \mathrm{CDM}$ simulations. A significant fraction of the final dark matter content of a cluster halo originates within galaxy-sized haloes, in which gas can cool and compress the dark matter core to high densities. Due to this adiabatic compression, the subsequent tidal stripping of the satellite dark matter should occur deeper within the cluster halo, depositing more mass near the cluster centre than would be expected without the compression operating within the satellites. As a result, the central mass profile of the galaxy cluster is substantially modified, even if the cooled galactic baryons are later redistributed throughout the cluster.

We note that Macciò et al. (2006) found in a simulation of a galactic halo that cooling produced a more highly concentrated dark matter profile for the host halo, but there, as in the cluster simulations, this may have been due directly to adiabatic compression in response to the large concentration of baryons in the centre of the host halo. Dolag et al. (2009) included radiative cooling and stellar feedback in simulations of cluster formation, and while they focused on the structure of the galactic subhaloes, they also found a $\sim 15$ per cent increase in the effective concentration of the cluster halo; this, however, may still be partly due to an increased baryon content near the cluster centre. Also, Sommer-Larsen \& Limousin (2009) artificially eliminated the overcooling problem in their cluster simulations and found only a very minor effect of the baryons on the total mass profile in this case; however, it is unclear what is the precise relation between our model and their particular feedback model and overcooling elimination scheme. One advantage of our analytical approach is that it allows us to cleanly separate the effect of density-enhanced tidal stripping from a simple overall adiabatic compression of the cluster halo. In general, the accuracy with which particular models capture tidal stripping could perhaps be checked by comparing with lensing probes of subhalo truncation in clusters (Limousin et al. 2009).

This paper is structured as follows. In Section 2, we develop a simple model for the adiabatic compression of satellites and for the subsequent tidal stripping of their dark matter within the cluster halo. We calculate in Section 3 the resulting final mass profile of the galaxy cluster, assuming that the cooled galactic baryons are later redistributed throughout the cluster. We show quantitatively that this can potentially explain the observed discrepancy. Finally, we summarize our conclusions and caveats in Section 4.

\section{MODEL}

We assume the standard $\Lambda$ CDM cosmology (Komatsu et al. 2009), with a dimensionless Hubble parameter $h=0.7$ and density parameters $\Omega_{\mathrm{m}}=0.28$ (dark matter plus baryons), $\Omega_{\Lambda}=0.72$ (cosmological constant) and $\Omega_{\mathrm{b}}=0.046$ (baryons). We also denote the cosmic baryon fraction by $f_{\mathrm{b}} \equiv\left(\Omega_{\mathrm{b}} / \Omega_{\mathrm{m}}\right)=0.16$.

Consider a halo that virialized at redshift $z$ in a flat $\Lambda \mathrm{CDM}$ universe. The critical density at $z$ is

$\rho_{\mathrm{c}}^{z}=\frac{3 H_{0}^{2}}{8 \pi G}\left[\Omega_{\mathrm{m}}(1+z)^{3}+\Omega_{\Lambda}\right]$

Numerical simulations of hierarchical halo formation indicate a roughly universal spherically averaged density profile for virialized haloes (Navarro, Frenk \& White 1997, hereafter NFW):

$\rho(r)=\rho_{\mathrm{c}}^{z} \frac{\delta_{\mathrm{c}}}{\frac{r}{r_{\mathrm{s}}}\left(1+\frac{r}{r_{\mathrm{s}}}\right)^{2}}$,

where the radius $r$ is divided by the scale radius $r_{\mathrm{s}}=r_{\mathrm{vir}} / c_{\mathrm{vir}}$ with $r_{\mathrm{vir}}$ being the virial radius, and the characteristic density $\delta_{\mathrm{c}}$ is related to the concentration parameter $c_{\mathrm{vir}}$ by

$\delta_{\mathrm{c}}=\frac{\Delta_{\mathrm{c}}}{3} \frac{c_{\mathrm{vir}}^{3}}{\ln \left(1+c_{\mathrm{vir}}\right)-c_{\mathrm{vir}} /\left(1+c_{\mathrm{vir}}\right)}$, 
where $\Delta_{\mathrm{c}}$ is the virial density in units of $\rho_{\mathrm{c}}^{z}$. For a halo of virial mass $M_{\text {vir }}$ at a given redshift $z$, the profile is fully specified by the parameters $\Delta_{\mathrm{c}}$ and $c_{\mathrm{vir}}$. We adopt the convention of a fixed $\Delta_{\mathrm{c}}=200$ at all redshifts, for consistency with the simulation analyses whose results we use.

We consider the stripping of a galactic satellite halo within a host cluster halo. We adopt a simple analytical model of tidal stripping that, in particular, has been previously used to understand how the density profiles of satellite subhaloes produce the central NFW profile of their final host halo (Syer \& White 1998). In this model, material originally at a radius $\xi$ within the satellite ends up, after tidal stripping, at a radius $r$ within the host halo so that the mean enclosed satellite density within $\xi$ equals the mean enclosed host density (before the stripping) within $r$ :

$\bar{\rho}_{\text {host }}^{0}(r)=\bar{\rho}_{\text {sat }}(\xi)$.

This condition corresponds to a resonance in dynamical frequencies between the circular orbit of a mass element at $\xi$ around the satellite and that of a circular satellite's orbit around the host halo, inducing an energy transfer that strips the mass element from the satellite. Setting the host tidal force equal to the internal gravitational force within the satellite would yield a very similar stripping radius. Note that this model assumes a simple picture in which incoming satellites follow circular orbits that slowly decay due to dynamical friction. More realistic models can try to improve on our approximate results by accounting for elongated orbits (see, e.g., Dekel, Devor \& Hetzroni 2003 for discussion).

We combine this stripping model with the simple model of adiabatic compression (Blumenthal et al. 1986), in which conservation of angular momentum implies that the quantity $r M(r)$ is fixed for each spherical mass shell, where $M(r)$ is the enclosed mass. We assume that both the host and each stripped satellite start out with NFW profiles. In satellites within the mass range of galaxies, the baryons cool and condense to the centre, inducing a change in the surrounding dark matter halo. Specifically, adiabatic compression moves a mass shell initially at $\xi_{\mathrm{i}}$, containing a mass $M_{\text {sat }}\left(\xi_{\mathrm{i}}\right)$, to a final radius $\xi_{\mathrm{f}}=\xi_{\mathrm{i}} M_{\text {sat }}\left(\xi_{\mathrm{i}}\right) / M_{\mathrm{f}}$, where the final enclosed mass is larger by a factor

$\frac{M_{\mathrm{f}}}{M_{\mathrm{sat}}\left(\xi_{\mathrm{i}}\right)}=1-f_{\mathrm{b}}+f_{\mathrm{b}} \frac{M_{\mathrm{sat}}}{M_{\mathrm{sat}}\left(\xi_{\mathrm{i}}\right)}$,

where $M_{\text {sat }}$ without an argument denotes the total virial mass of the satellite. The stripping model then implies that the same mass shell ends up at a radius $r_{\mathrm{f}}$ in the host cluster halo, where

$\bar{\rho}_{\text {host }}^{0}\left(r_{\mathrm{f}}\right)=\bar{\rho}_{\text {sat }}\left(\xi_{\mathrm{i}}\right) \times\left[\frac{M_{\mathrm{f}}}{M_{\text {sat }}\left(\xi_{\mathrm{i}}\right)}\right]^{4}$

in terms of the initial enclosed density $\bar{\rho}_{\text {sat }}\left(\xi_{\mathrm{i}}\right)$ in the satellite (i.e. before the adiabatic compression). The power of four on the righthand side results from the increased mass (one power) and decreased radius (hence three powers in the density). Note that the cooled baryonic cores of the galaxies are much denser than their surrounding dark matter haloes and so we have assumed that the baryonic cores are not tidally stripped.

In deriving equation (5) we have assumed that in galactic haloes, where the cooling time of the virialized gas is much shorter than the Hubble time, the full baryonic content of the haloes initially cools toward the centre of the halo, condensing the surrounding dark matter before it gets stripped. Observationally, even the fraction of galactic halo baryons that are in stars today is not well known, since the hot baryons in haloes are difficult to detect, while total masses of galactic haloes are difficult to measure accurately (and can be used to obtain the total gas mass only with the added assumption of a halo baryon fraction that equals the cosmic mean). The best estimate for the total mass of stars and stellar remnants today, as a fraction of the total baryonic mass that lies within virialized regions of galaxies, is $\sim 10$ per cent, with an uncertainty of order 50 per cent (Fukugita \& Peebles 2004). For our own Milky Way Galaxy, the disc and bulge may make up as much as 40 per cent of the halo baryons (Xue et al. 2008). Regardless of the precise fraction today, it is plausible to assume that most of the gas in galaxies initially cooled and was later expelled over time from the central region back into the halo, through supernova or quasar feedback. Thus, we expect that if galactic haloes were stripped within the cluster relatively early, then star formation and feedback did not have much time to operate prior to the stripping. This scenario does not conflict with the fact that clusters only virialized relatively recently, since we are focusing here on the stripping that formed the inner regions of clusters, within a tenth of the virial radius, and this likely occurred long before the entire cluster virialized.

In clusters, the originally cooled gas must eventually have gotten dispersed into the intracluster medium (ICM), since observations find only a small fraction of cluster baryons residing near the centre. In the cluster environment, this gas redistribution can be facilitated by interactions among galaxies or with the ICM, in addition to internal galactic feedback. For simplicity, we assume that the final baryon distribution is similar to that of the dark matter, i.e. the final baryon fraction is uniform and equal to the cosmic value. Within our model above, this effectively means that the satellites contribute only $M_{\text {sat }}\left(\xi_{\mathrm{i}}\right)$ to the mass enclosed within the final cluster radius $r_{\mathrm{f}}$ (and not the full $M_{\mathrm{f}}$ ).

In this picture, when the cooled galactic baryons get redistributed throughout the final cluster halo, they may cause a partial adiabatic expansion of the halo. However, this should roughly cancel the initial adiabatic contraction of the surrounding halo material when the satellites enter and are stripped; thus, we neglect both the initial halo contraction and the later expansion, as we do not expect a significant net effect. This is different from the main process that we focus on, where the early adiabatic compression causes the satellites' dark matter to be stripped at smaller cluster radii than it would otherwise, leaving a net effect on the cluster in the end.

The stripping model that led to equation (6) assumes that the host halo is dominant, and that the satellites contribute only a small fraction of the enclosed mass at the stripping radius. This assumption breaks down, however, when the satellites dominate, and we do expect such a regime; indeed, it is plausible (and indicated by pure dark matter simulations) that the dense core of the cluster halo arises entirely from the original cores of the accreted satellites, since only lower density material is accreted later on to the cluster halo (Loeb \& Peebles 2003; Gao et al. 2004). In this high-density regime where the satellites dominate relative to the pre-existing halo material, we expect the satellite cores to simply settle in the host core, preserving in the end their original densities, since they first adiabatically compress and later re-expand. This limit is consistent with the equal-density relation in equation (4); while this equation was originally derived for stripping on to a dominant host halo, we can use it to capture the dominant satellite limit, if we obtain from it the radius $r_{\mathrm{f}}$ at which the satellite mass ended up (reinterpreted as contributing part of the halo profile rather than adding mass on top of $\left.\bar{\rho}_{\text {host }}^{0}\right)$. We leave for future work a detailed analysis of the complex transition region between the two limits of equation (4) (density preservation) and equation (6) (density enhancement), and here we adopt a simple interpolation between them. This is reasonable given our limited goal of examining whether the halo profile can be 
substantially modified at all. Thus, we determine the stripping radius by solving (for a given $\xi_{\mathrm{i}}$ )

$\bar{\rho}_{\text {host }}^{0}\left(r_{\mathrm{f}}\right)=\bar{\rho}_{\text {sat }}\left(\xi_{\mathrm{i}}\right) \times\left\{f_{\text {sat }}\left(r_{\mathrm{f}}\right)+\left[1-f_{\text {sat }}\left(r_{\mathrm{f}}\right)\right]\left[\frac{M_{\mathrm{f}}}{M_{\text {sat }}\left(\xi_{\mathrm{i}}\right)}\right]^{4}\right\}$,

where $f_{\text {sat }}\left(r_{\mathrm{f}}\right)$ is the fractional contribution of the satellites to the enclosed mass within the stripping radius $r_{\mathrm{f}}$, and the right-hand side of this equation interpolates between equation (4) [valid in the limit $f_{\text {sat }}\left(r_{\mathrm{f}}\right)=1$ ] and equation (6) [valid when $f_{\text {sat }}\left(r_{\mathrm{f}}\right)=0$ ].

In order to solve the model, we must determine various masses. First, based on the host NFW profile in the absence of cooling and adiabatic compression, we obtain the enclosed host mass at each radius, $M_{\text {host }}^{0}\left(r_{\mathrm{f}}\right)=(4 / 3) \pi r_{\mathrm{f}}^{3} \bar{\rho}_{\text {host }}^{0}\left(r_{\mathrm{f}}\right)$. We use equation (4) to calculate the fractional contribution of the satellites to this enclosed mass in the absence of cooling, $f_{\mathrm{sat}}^{0}\left(r_{\mathrm{f}}\right)$. Adiabatic compression and stripping then replaces the satellite contribution by $M_{\mathrm{f}}$, and thus in equation (7) we set

$f_{\text {sat }}\left(r_{\mathrm{f}}\right)=\frac{M_{\mathrm{f}}}{\left[1-f_{\text {sat }}^{0}\left(r_{\mathrm{f}}\right)\right] M_{\text {host }}^{0}\left(r_{\mathrm{f}}\right)+M_{\mathrm{f}}}$.

After solving for $r_{\mathrm{f}}$, the final enclosed mass within this radius, after feedback redistributes the baryons, is

$M_{\text {host }}\left(r_{\mathrm{f}}\right)=\left[1-f_{\text {sat }}^{0}\left(r_{\mathrm{f}}\right)\right] M_{\text {host }}^{0}\left(r_{\mathrm{f}}\right)+M_{\text {sat }}\left(\xi_{\mathrm{i}}\right)$.

Within our simple model, the redistribution of mass depends only on the density profile of the satellites, i.e. on the functional form of $M_{\text {sat }}\left(\xi_{\mathrm{i}}\right) / M_{\text {sat }}$ versus $\bar{\rho}_{\text {sat }}\left(\xi_{\mathrm{i}}\right)$, and not on the number of satellites or their individual total masses. Since we are interested in the galactic subhaloes that end up in the cluster, we can adopt the typical value of accretion redshift $z_{\text {sat }}$ and NFW concentration $c_{\text {vir }}$ for such haloes, and effectively calculate stripping of one satellite that contributes some total fraction $f_{\text {sat }}$ of the host cluster mass. Within the model, this single satellite represents the cumulative effect of all the individual galactic satellites that merged into the final cluster halo.

In order to quantify the effect of compressed galactic haloes, we must estimate the effective $f_{\text {sat }}$, i.e. the fraction of cluster dark matter that arrived from within galactic haloes in which the baryons were able to cool. We can obtain a theoretical estimate for the fraction of cluster dark matter that passed in its merger history through galactic haloes, on the way to becoming part of the final cluster halo. For concreteness, let us consider the progenitor distribution at various redshifts of a $10^{15} \mathrm{M}_{\odot}$ cluster halo at $z \sim 0.2-0.4$. The extended Press-Schechter model (Bond et al. 1991) then implies that $\sim 25$ per cent of the cluster mass was in haloes with masses in the range $10^{10}-10^{12} \mathrm{M}_{\odot}$ at $z \sim 2.5$ (the redshift that maximizes this fraction). A full merger tree would give on average at least this value since additional cluster mass that was outside this halo mass range at $z=2.5$ may have passed through galactic haloes at other redshifts.

We can also estimate the fraction $f_{\text {sat }}$ from observations. A first attempt might proceed as follows. Stars make up $\sim 1$ per cent of the total virialized mass of massive clusters (e.g. Lagana et al. 2008), which corresponds to $\sim 5$ per cent of the total baryonic mass. To find the baryon fraction that was associated with the galaxies in which these stars formed, we must divide by their average star formation efficiency. As noted above, the total stellar mass today is $\sim 10$ per cent of the baryon mass within virialized regions of galaxies (Fukugita \& Peebles 2004). This suggests that $\sim 50$ per cent of the cluster gas was processed through galaxies, and thus also a similar fraction of the cluster's dark matter was contributed by stripped satellite galaxies (assuming that the total baryon fraction of both these galaxies and the final cluster is equal to the cosmic mean fraction). However, this estimate depends on the uncertain value of the star formation efficiency. We can use metallicity measurements to obtain a more direct estimate of $f_{\text {sat }}$. The typical metallicity of the ICM in clusters at redshift $z \sim 0.3$ is $0.3-0.4$ of the solar abundance (e.g. Maughan et al. 2008), while massive galaxies (in clusters or the field) typically have a solar abundance or less (e.g. Ellison et al. 2009), indicating that at least 30-40 per cent of the cluster gas must have been processed in galaxies in order for the ICM to reach its high metallicity value. Within our model, higher $f_{\text {sat }}$ values lead to more highly concentrated cluster haloes (see the next section). Taking into account these various considerations, we consider $f_{\text {sat }}$ values in the range $20-40$ per cent.

We note that Gnedin et al. (2004) showed that the classic adiabatic contraction model that we use tends to overestimate the effect of a central baryon concentration on dark matter, compared to simulated profiles. The overestimate in the mass profile, however, is under 10 per cent at the radii that we focus on $\left(\sim 0.1 r_{\text {vir }}\right)$, thus justifying our use of the simple model. The overestimate does increase at smaller radii and is $\sim 50$ per cent at $0.01 r_{\text {vir }}$, implying that the profiles we find below are less reliable in the innermost region.

\section{RESULTS}

In this section, we quantify the effect that adiabatic compression in galactic satellites can have on the final density profile of the host cluster halo. We compare our results to the four clusters considered by Broadhurst \& Barkana (2008), and also make use of their results for the theoretical predictions. In particular, we adopt the NFW parameters measured by Neto et al. (2007) for simulated haloes, after correcting them based on Hennawi et al. (2007) to obtain the effective parameters for the population of lensing clusters, observed in projection; however, we reduce $c_{\mathrm{vir}}$ by 10 per cent according to the recent results of Duffy et al. (2008). This yields a median $c_{\mathrm{vir}}=5.5$ for the most massive clusters, with a $1 \sigma$ range (for the effective projected $c_{\mathrm{vir}}$ ) of 4-7.5 (approximately in a lognormal distribution). Studies based on large numerical simulations (Zhao et al. 2003; Gao et al. 2008) have found for massive haloes a relatively weak decline of $c_{\text {vir }}$ with increasing redshift, but a more significant decline for galactic mass haloes, with $c_{\text {vir }} \sim 4$ for $\sim 10^{11}-10^{12} \mathrm{M}_{\odot}$ haloes at $z \sim 2$. In our quantitative results, we adopt $c_{\mathrm{vir}}=4$ and $z_{\text {sat }}=$ 2 for the satellites, and consider hosts at $z=0.3$ with various concentration parameters. As noted above, we assume that the dense core of the host arises entirely from the satellite cores, so for each host $c_{\text {vir }}$ this normalizes the total satellite fraction $f_{\text {sat }}$ of the cluster's virial mass. In particular, we consider $f_{\text {sat }}=20$ per cent (which implies a host $\left.c_{\mathrm{vir}}=6.8\right), f_{\mathrm{sat}}=30$ per cent $\left(c_{\mathrm{vir}}=7.5\right)$, or $f_{\text {sat }}=40$ per cent $\left(c_{\text {vir }}=8.0\right)$. These host concentration parameters are somewhat high but still near or within the expected $1 \sigma$ range for cluster haloes, as noted above.

Fig. 1 shows the effect of adiabatic compression on the 3D mass profiles of clusters. The effect is largest at the innermost radii, where the satellites contribute a substantial fraction of the halo mass. This is true even though our model in equation (7) suppresses the density enhancement in this $f_{\text {sat }}\left(r_{\mathrm{f}}\right) \rightarrow 1$ limit; since the enclosed mass changes rapidly with radius in the core, even a slight shift in the stripping radius has a large effect on the mass profile. For the cases considered, where the fractional mass contribution by satellites $f_{\text {sat }}$ is 20,30 or 40 per cent, we find that the cluster profile at $r \lesssim 0.1 r_{\text {vir }}$ is substantially modified by adiabatic compression in the satellites, even though the baryonic mass is assumed to have been redistributed 


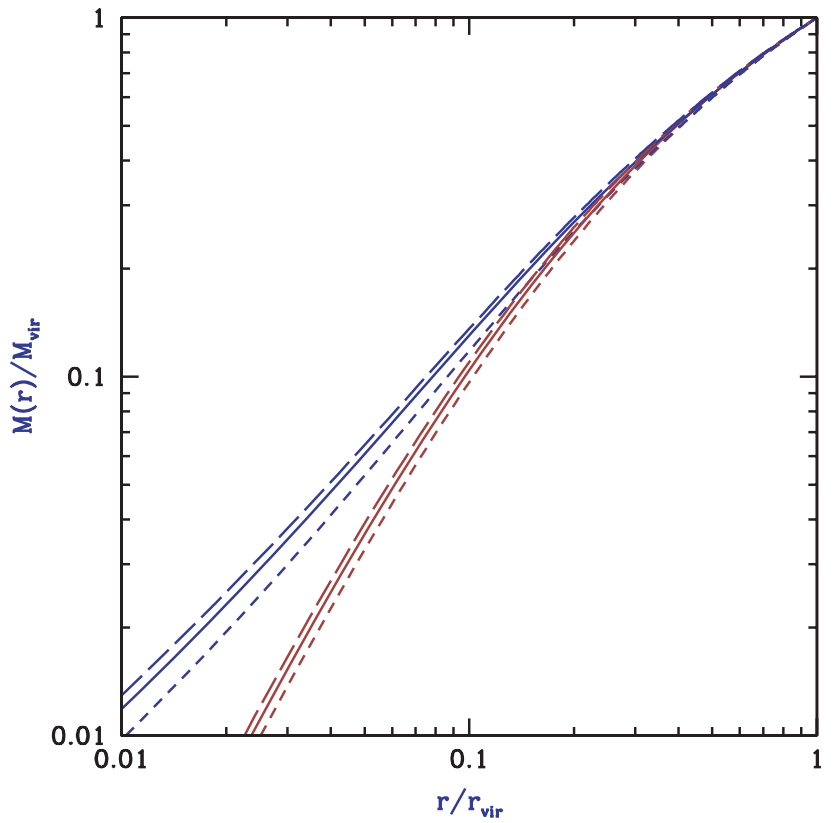

Figure 1. Profiles of the enclosed 3D mass in clusters as a function of radius. We consider a host halo that follows an NFW model at $z=0.3$, and assume satellites with $c_{\text {vir }}=4$ at redshift $z_{\text {sat }}=2$ that make up a total fraction $f_{\text {sat }}$ of the cluster's virial mass. We consider a host $c_{\mathrm{vir}}=6.8$ and $f_{\text {sat }}=20$ per cent (short-dashed curves), a host $c_{\mathrm{vir}}=7.5$ and $f_{\text {sat }}=30$ per cent (solid curves), or a host $c_{\text {vir }}=8.0$ and $f_{\text {sat }}=40$ per cent (long-dashed curves). In each case, we show the host profile in the absence of cooling and adiabatic compression (bottom curve), and the final profile (top curve) of a host that accreted satellites that underwent gas cooling, adiabatic compression and stripping of their dark matter haloes before their baryons were redistributed throughout the cluster. In each case, $f_{\text {sat }}$ is normalized so that the host's dense core arises entirely from the satellite cores.

uniformly after the stripping. The enclosed mass at $r=0.1 r_{\text {vir }}$ is increased by 22 per cent (for $f_{\text {sat }}=20$ per cent), 26 per cent (for $f_{\text {sat }}=30$ per cent), or 24 per cent (for $f_{\text {sat }}=40$ per cent), and this enhancement factor grows rapidly towards smaller radii.

The model's features are illustrated in Fig. 2, which shows the fractional satellite contribution to the halo mass profile. In each case, the fraction is fixed to $f_{\text {sat }}$ at the virial radius and unity at $r \rightarrow 0$. There is a break in the curves at $r=0.44 r_{\text {vir }}$, which is the maximum cluster radius that can receive a contribution from the satellite haloes. This maximum arises from the higher virial density at $z=2$ compared to the corresponding value at $z=0.3$. At each radius, the satellite mass fraction reaches its highest value during stripping, when it enjoys the enhancement due to adiabatic compression but does not yet suffer the reduction due to the redistribution of baryons. At $r=0.1 r_{\text {vir }}$, this fraction is between 60 and 90 per cent, which makes our results at this radius (as seen in Fig. 1 and below) relatively insensitive to the precise value of $f_{\text {sat }}$; higher values of $f_{\text {sat }}$ push the satellite fraction higher towards unity, and in this limit stripping cannot significantly increase the density of the satellite's dark matter, as reflected in equation (7).

The implications of adiabatic compression for gravitational lensing are displayed in Fig. 3, which shows the profile of the enclosed, projected 2D mass density. We consider the same cluster and satellite halo parameters as in the previous figures, and focus on the range of projected radius corresponding to observed cluster Einstein radii. For each of the observed clusters, we show the critical lensing density versus effective Einstein radius, as a central point

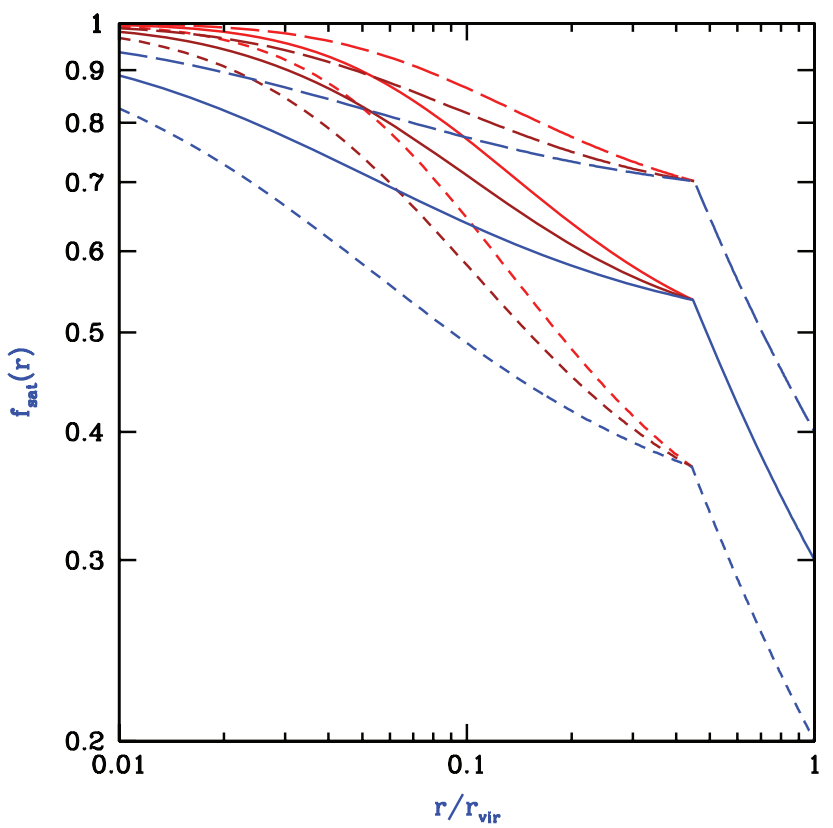

Figure 2. Radial profile of the fractional satellite contribution to the enclosed halo mass. Assumptions and notations are the same as in Fig. 1, and in particular we consider $f_{\text {sat }}=20$ per cent (short-dashed curves), 30 per cent (solid curves), or 40 per cent (long-dashed curves). In each case, we show the satellite fraction in the absence of cooling and adiabatic compression [ $f_{\text {sat }}^{0}(r)$, bottom curve], the final fraction after stripping and baryon redistribution (based on equation 9 , middle curve) and the higher fraction present during stripping but still before baryon redistribution (equation 8 , top curve).

plus $1 \sigma$ error ellipse. The figure is consistent with the factor of $\sim 2$ discrepancy highlighted by Broadhurst \& Barkana (2008) between the observed $r_{\mathrm{E}}$ and the median theoretical prediction from pure dark matter simulations; here the typical value of $r / r_{\text {vir }}$ (for the bottom curves) at the $\Sigma_{\mathrm{cr}}$ observed for each cluster is smaller only by a factor of $\sim 1.5$, since our model has required us to adopt somewhat higher than average cluster halo concentrations. Still, these predictions in the absence of baryonic cooling for the most part lie well outside the $1 \sigma$ error ellipses of the observed clusters.

With the natural parameters that we have assumed for the satellites, adiabatic compression resolves the current discrepancy; it boosts the theoretical predictions enough to bring them well within the observed error ellipses. For example, if $f_{\text {sat }}=30$ per cent then for A1689, the observed (central) value $r / r_{\text {vir }}=0.072$ for the effective Einstein radius can be compared with the predicted value (at the same projected surface density equal to the critical lensing density) of $r / r_{\text {vir }}=0.064$ (with adiabatic compression in the satellites), and the previous pure dark matter prediction (i.e. without adiabatic compression) of $r / r_{\text {vir }}=0.045$. For A1703, the observed $r / r_{\text {vir }}=0.070$ can be compared with the theoretical $r / r_{\text {vir }}=0.070$ (with adiabatic compression) and $r / r_{\mathrm{vir}}=0.052$ (without). For Cl0024-17, the corresponding numbers are $r / r_{\text {vir }}=0.100$ compared to $r / r_{\mathrm{vir}}=0.081$ (with) and $r / r_{\mathrm{vir}}=0.064$ (without); for RX J1347, $r / r_{\text {vir }}=0.109$ compared to $r / r_{\text {vir }}=0.100$ (with) and $r / r_{\text {vir }}=0.086$ (without). While the observed clusters still have slightly high Einstein radii compared to the typical expected cluster profile, the theoretical scatter in $c_{\text {vir }}$ together with the observational errors make the theoretical and observational predictions consistent with each other. 


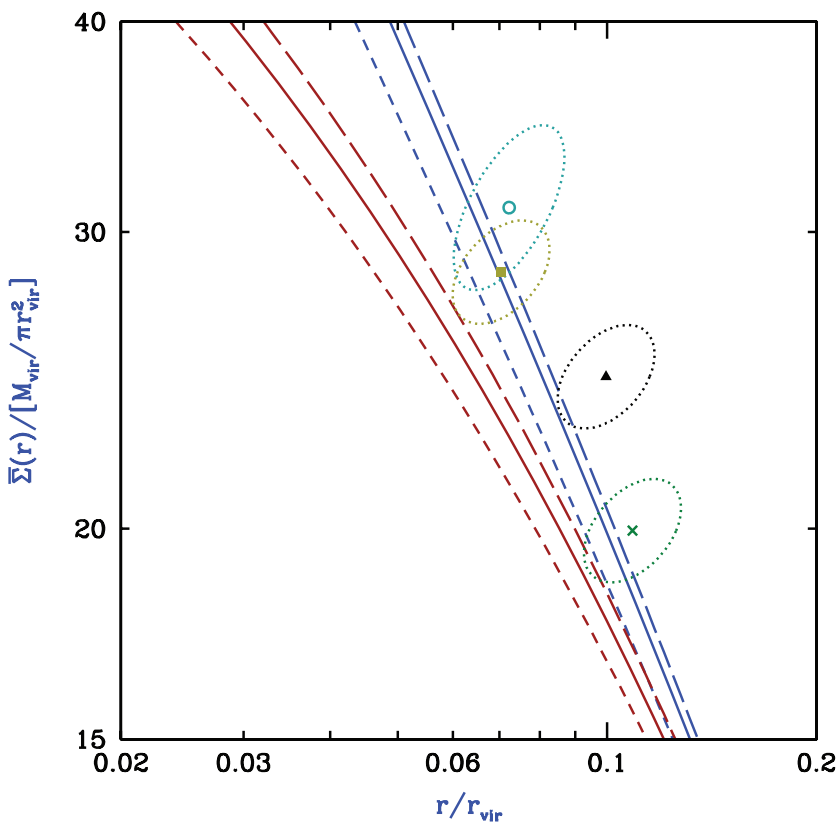

Figure 3. Profile of the enclosed projected surface mass density versus projected radius. Assumptions and notations are the same as in Fig. 1, and in particular we consider $f_{\text {sat }}=20$ per cent (short-dashed curves), 30 per cent (solid curves), or 40 per cent (long-dashed curves). For comparison, we show the observed values for four clusters, A1689 (open circle), A1703 (square), Cl0024 (triangle) and RX J1347 (×). For each cluster, we show its critical density for lensing versus effective Einstein radius, with the dot indicating a central location and the ellipse showing the combined $1 \sigma$ uncertainties due to the measurement errors in $r_{\mathrm{E}}$ and $M_{\mathrm{vir}}$.

As we have shown, our results depend only weakly on $f_{\text {sat }}$, as long as it is within a reasonable range. The results also depend slightly on other assumed properties of the satellites. We illustrate this for A1689, fixing $f_{\text {sat }}=30$ per cent and adjusting the host concentration accordingly in each case. We find that lowering the satellite $c_{\text {vir }}$ to 3 at $z_{\text {sat }}=2$ decreases the predicted $r / r_{\text {vir }}$ by 14 per cent, while raising $c_{\text {vir }}$ to 5 increases it by 12 per cent. Assuming $c_{\mathrm{vir}}=3$ at $z_{\mathrm{sat}}=3$ raises the predicted $r / r_{\mathrm{vir}}$ by 3 per cent, while $c_{\mathrm{vir}}=5$ at $z_{\mathrm{sat}}=1$ lowers it by 13 per cent, all compared to our standard case of $c_{\mathrm{vir}}=4$ at $z_{\mathrm{sat}}=2$. Finally, if we assume that only 50 per cent (rather than 100 per cent) of the baryons in the galactic satellites cooled and condensed before their haloes were stripped, i.e. in equation (5) we use half the cosmic fraction for $f_{\mathrm{b}}$, then the predicted $r / r_{\text {vir }}$ is reduced by 9 per cent for A1689 and $f_{\text {sat }}=30$ per cent.

\section{DISCUSSION}

We have demonstrated that dark matter compression due to baryonic cooling inside galaxy haloes can in turn leads to tidal stripping of these galactic haloes closer to the centre of the galaxy cluster in which they reside. Even if the baryons are later redistributed within the cluster by feedback, a substantial effect remains due to the early adiabatic compression. This effect can explain the high central mass concentration of clusters in lensing observations. Our scenario, in which only the inner 10-20 per cent of the virial radius is significantly modified, is consistent with weak lensing measurements at larger radii that find low cluster halo concentrations (e.g. Mandelbaum, Seljak \& Hirata 2008).
We have adopted a number of simplifying approximations in showing the existence of the effect. Hydrodynamical simulations that avoid overcooling of the baryons at the cluster core are necessary in order to test our proposed mechanism in quantitative detail. Nevertheless, our simplified treatment has demonstrated the general point that it is possible for gas physics to significantly change the Einstein radius of massive clusters, even without leaving a central baryon concentration.

Finally, we note that ram pressure stripping of hot accreted baryons, which make up the majority ( $\sim 80$ per cent $)$ of the cluster baryons, may help to reduce the central baryon fraction. Indeed, cluster simulations find a reduced baryon fraction at $r / r_{\text {vir }} \lesssim 0.2$ (e.g. Kravtsov et al. 2005; Dolag et al. 2009) and match better the baryonic fraction inferred from X-ray observations (Vikhlinin et al. 2009).

\section{ACKNOWLEDGMENTS}

$\mathrm{RB}$ is grateful for the kind hospitality of the Institute for Theory and Computation (ITC) at the Harvard-Smithsonian CfA, and also acknowledges support by the Moore Distinguished Scholar programme at Caltech and the John Simon Guggenheim Memorial Foundation. This work was supported in part by NASA grant NNX08AL43G, by Harvard University funds and by BSF grant 2004386.

\section{REFERENCES}

Afshordi N., Lin Y.-T., Nagai D., Sanderson A. J. R., 2007, MNRAS, 378 293

Astier P. et al., 2006, A\&A, 447, 31

Blumenthal G. R., Faber S. M., Flores R., Primack J. R., 1986, ApJ, 301, 27

Bond J. R., Cole S., Efstathiou G., Kaiser N., 1991, ApJ, 379, 440

Bradač M. et al., 2008, ApJ, 681, 187

Broadhurst T., Barkana R., 2008, MNRAS, 390, 1647

Broadhurst T., Takada M., Umetsu K., Kong X., Arimoto N., Chiba M., Futamase T., 2005, ApJ, 619, L143

Dekel A., Devor J., Hetzroni G., 2003, MNRAS, 341, 326

Dolag K., Borgani S., Murante G., Springel V., 2009, MNRAS, 399, 497

Duffy A. R., Schaye J., Kay S. T., Dalla Vecchia C., 2008, MNRAS, 390, L64

Ellison S. L., Simard L., Cowan N. B., Baldry I. K., Patton D. R., McConnachie A. W., 2009, MNRAS, 396, 1257

Fukugita M., Peebles P. J. E., 2004, ApJ, 616, 643

Gao L., Loeb A., Peebles P. J. E., White S. D. M., Jenkins A., 2004, ApJ, 614, 17

Gao L., Navarro J. F., Cole S., Frenk C. S., White S. D. M., Springel V., Jenkins A., Neto A. F., 2008, MNRAS, 387, 536

Gavazzi R., Fort B., Mellier Y., Pelló R., Dantel-Fort M., 2003, A\&A, 403, 11

Gnedin O. Y., Kravtsov A. V., Klypin A. A., Nagai D., 2004, ApJ, 616, 16 Halkola A., Hildebrandt H., Schrabback T., Lombardi M., Bradac M., Erben T., Schneider P., Wuttke D., 2008, A\&A, 481, 65

Hennawi J. F., Dalal N., Bode P., Ostriker J. P., 2007, ApJ, 654, 714

Kling T. P., Dell'Antonio I., Wittman D., Tyson J. A., 2005, ApJ, 625, 643

Kneib J.-P.et al., 2003, ApJ, 598, 804

Komatsu E. et al., 2009, ApJS, 180, 330

Kravtsov A. V., Nagai D., Vikhlinin A. A., 2005, ApJ, 625, 588

Lagana T. F., Lima Neto G. B., Andrade-Santos F., Cypriano E. S., 2008, A\&A, 485, 633

LaRoque S. J., Bonamente M., Carlstrom J. E., Joy M. K., Nagai D., Reese E. D., Dawson K. S., 2006, ApJ, 652, 917

Lemze D., Barkana R., Broadhurst T. J., Rephaeli Y., 2008, MNRAS, 386, 1092

Limousin M. et al., 2007, ApJ, 668, 643 
Limousin M., Sommer-Larsen J., Natarajan P., Milvang-Jensen B., 2009, ApJ, 696, 1771

Lin Y.-T., Mohr J. J., Stanford S. A., 2004, ApJ, 610, 745

Loeb A., Peebles P. J. E., 2003, ApJ, 589, 29

Macciò A. V., Moore B., Stadel J., Diemand J., 2006, MNRAS, 366, 1529

Mandelbaum R., Seljak U., Hirata C. M., 2008, J. Cosmology Astroparticle Phys., 08, 006

Maughan B. J., Jones C., Forman W., Van Speybroeck L., 2008, ApJS, 174, 117

Navarro J. F., Frenk C. S., White S. D. M., 1997, ApJ, 490, 493 (NFW)

Neto A. F. et al., 2007, MNRAS, 381, 1450

Oguri M., Blandford R. D., 2009, MNRAS, 392, 930

Oguri M. et al., 2009, ApJ, 699, 1038

Percival W. J., Cole S., Eisenstein D. J., Nichol R. C., Peacock J. A., Pope A. C., Szalay A. S., 2007, MNRAS, 381, 1053
Rozo E., Nagai D., Keeton C., Kravtsov A., 2008, ApJ, 687, 22

Sommer-Larsen J., Limousin M., 2009, MNRAS, submitted (arXiv: 0906.0573)

Syer D., White S. D. M., 1998, MNRAS, 293, 337

Umetsu K., Broadhurst T., 2008, ApJ, 684, 177

Vikhlinin A. et al., 2009, ApJ, 692, 1033

Xue X.-X.et al., 2008, ApJ, 684, 1143

Zhao D. H., Jing Y. P., Mo H. J., Börner G., 2003, ApJ, 597, L9

Zitrin A., Broadhurst T., 2009, ApJ, 703, 132

This paper has been typeset from a $\mathrm{T}_{\mathrm{E}} \mathrm{X} / \mathrm{LAT}_{\mathrm{E}} \mathrm{X}$ file prepared by the author. 